\title{
Anatomía ecocardiográfica de un mixoma neovascularizado
}

\section{Edgar Fuentes-Molina}

Especialista en Cardiología. Máster en Ecocardiografía Transesofágica. Fellow de Ecocardiografía SISIAC. Profesor de Posgrado de Cardiología (Universidad de Costa Rica). Jefe del Laboratorio de Ecocardiografía del Hospital San Juan de Dios. San José. Costa Rica

Recibido: 29/05/2020

Aceptado: 29/05/2020

En línea: 31/07/2020

Citar como: Fuentes-Molina E. Anatomía ecocardiográfica de un mixoma neovascularizado. Rev Ecocar Pract (RETIC). 2020 (Jul); 3 (2): 26-28. doi: 10.37615/retic. v3n2a8.

Cite this as: Fuentes-Molina E. Echocardiographic anatomy of a neo-vascularized myxoma. Rev Ecocar Pract (RETIC). 2020 (Jul); 3 (2): 26-28. doi: 10.37615/retic. v3n2a8.

\section{Palabras clave}

$\triangleright$ Mixoma

$\triangleright$ 3D

$\triangleright$ Ecocardiografía

\section{RESUMEN}

Clásicamente, el diagnóstico de mixoma cardíaco está basado principal y fundamentalmente en la ecocardiografía transtorácica y transesofágica, y en general no ofrece mayor dificultad si la presentación es típica; sin embargo, en no pocas ocasiones, se suelen utilizar otras modalidades de imagen para "confirmar" o "asegurar" el diagnóstico, sin lograr agotar antes todas las opciones que ofrece la ecocardiografía hoy en día, tanto con técnicas novedosas, como con técnicas antiguas que muchas veces se dejan de lado. Se presenta un caso típico de mixoma, con algunos hallazgos ecocardiográficos que van más allá del simple hallazgo de una masa cardíaca.

\section{Presentación del caso}

Varón de 77 años con sobrepeso, dislipidemia e hipertensión arterial, sin antecedentes cardiovasculares previos con un cuadro de 4 meses de disnea de moderados esfuerzos, astenia, adinamia y fatiga. En el transcurso de ese tiempo fue valorado por su médico general y el médico internista, y se le realizaron algunos estudios básicos, incluyendo una radiografía de tórax sin cardiomegalia ni congestión venocapilar, un electrocardiograma en ritmo sinusal sin hallazgos sugestivos de isquemia previa, un hemograma normal y un NT-pro-BNP de $450 \mathrm{pg} / \mathrm{ml}$

Tras esta evaluación inicial, se decide realizar una ecocardiografía transtorácica, que documentó como hallazgo principal la presencia de una masa redondeada en la aurícula izquierda adyacente al septum interauricular, con lo que se plantea como primera opción diagnóstica el mixoma. Ante este hallazgo se realiza una ecocardiografía transesofágica. La Figura 1 muestra la presencia de una masa redondeada de $30 \times 29 \mathrm{~mm}$ localizada en la aurícula izquierda, adyacente y anexa al septum interauricular, mientras que el Vídeo 1 permite ver la relativamente escasa movilidad de la masa, y a la vez el comportamiento dinámico de la misma, que impresiona elongarse y contraerse durante el ciclo cardíaco.
La ecocardiografía transesofágica tridimensional permite una valoración detallada de la masa, así como de su ubicación espacial y relación con estructuras adyacentes, tal como se ve en el Vídeo 2 y en el Vídeo 3. En el Vídeo 4 se puede observar una adquisición en modalidad de volumen completo de aurícula y ventrículo izquierdos, en la que se aprecia la masa auricular y su relación con la válvula mitral. Además, se ha disminuido la ganancia 2D/4D intencionalmente, con el fin de "hacer desaparecer" el tejido superficial de la masa, y dejar en evidencia el esqueleto fibroso de la misma, hallazgo que apoya la naturaleza tumoral de la masa. Este manejo de las ganancias es el responsable de los múltiples drop-outs en el resto de la imagen. La adquisición tridimensional de volumen completo permite, a su vez, "disecar" ese volumen y "cortarlo" en múltiples cortes tomográficos; esto es lo que se observa en el Vídeo 5, en el que se observan múltiples cortes tomográficos de la masa, en sentido lateralmedial, y posterior-anterior. Tal grado de "escudriñamiento" del interior de la masa permite visualizar ahora con mayor claridad el grado de heterogeneidad en su interior, lo que podría corresponder a tipos distintos de tejidos, y/o un mismo tejido en diferentes estados evolutivos (necrosis, edema, infiltración leucocitaria...).

En el Vídeo 6 se puede observar otra técnica ecocardiográfica que también es útil en la evaluación de masas cardíacas y que permite discernir entre tumor frente a trombo intracardíaco: la visualización de flujo sanguíneo en el interior 
de la masa. Para ello, y de manera análoga a como se estima la reserva coronaria de la arteria descendente anterior de forma no invasiva, la idea es localizar alguno de los vasos nutricios de la masa, mediante la disminución de la escala de Nyquist del Doppler color (en este caso a $16 \mathrm{~cm} / \mathrm{s}$ ), y optimizando la ganancia. Es posible observar dicho flujo con claridad con su correspondiente Doppler espectral (Figura 2), que confirma su origen coronario.

El paciente fue remitido a Cirugía Cardíaca para resección de la masa en un centro terciario. Histológicamente se demostró que era un mixoma.

\section{Estudio por imagen}

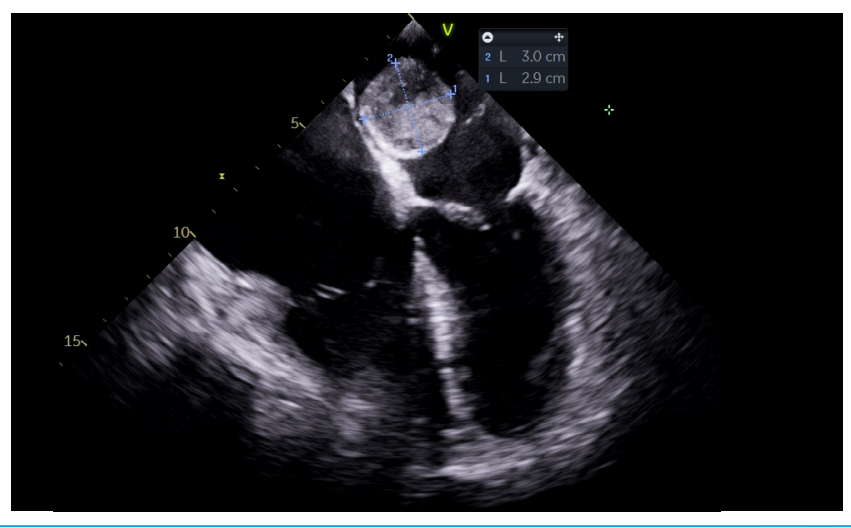

Figura 1. ETE. Vista apical de cuatro cámaras medioesofágico. Masa de $30 \mathrm{x}$ $29 \mathrm{~mm}$ redondeada en aurícula izquierda, adosada al septum interauricular

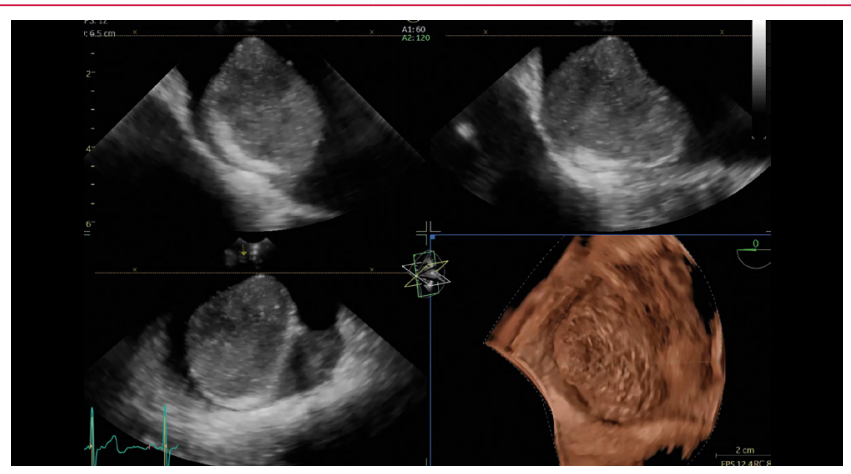

Vídeo 1. ETE. Secuencia combinada de zoom multiplanar e imagen volumétrica "renderizada" de la masa; se logra visualizar que la masa no es homogénea, tiene una cápsula hiperecogénica y, además, la base de implantación es ancha (cuadrante inferior izquierdo)

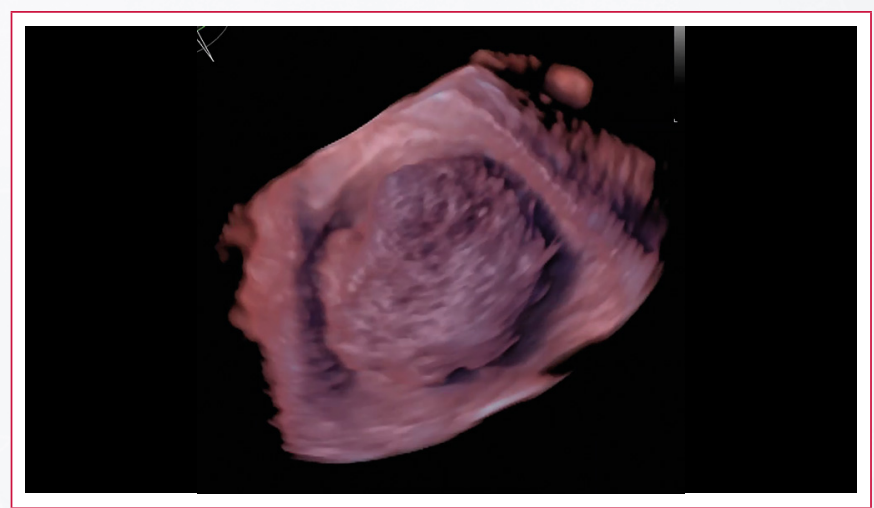

Vídeo 2. ETE. Zoom tridimensional de la masa en aurícula izquierda

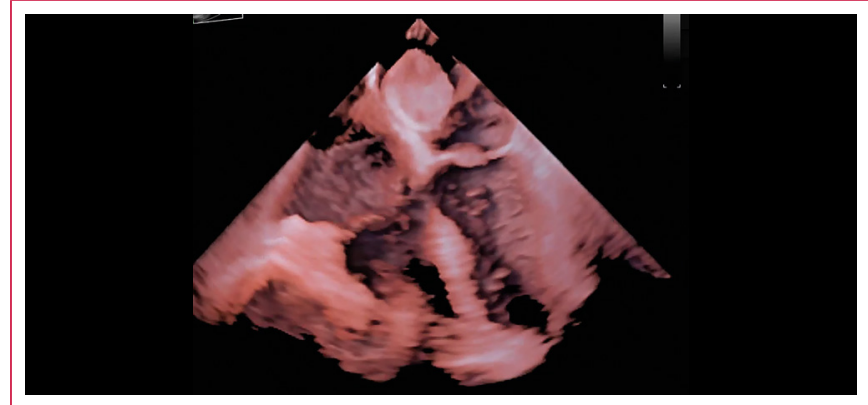

Vídeo 3. ETE tridimensional en tiempo real, vista apical de cuatro cámaras Se aprecia, con una mayor panorámica, la ubicación espacial de la masa, as como la relación con el resto de las cámaras cardíacas

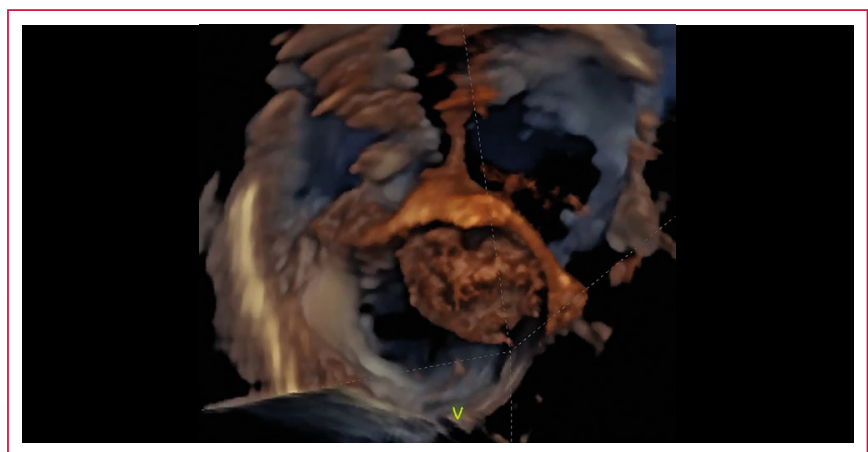

Vídeo 4. ETE. Volumen completo tridimensional. Se visualiza la masa en aurícula izquierda, y su relación con la válvula mitral y el septum interauricular. Nótese el manejo de la ganancia 2D/4D

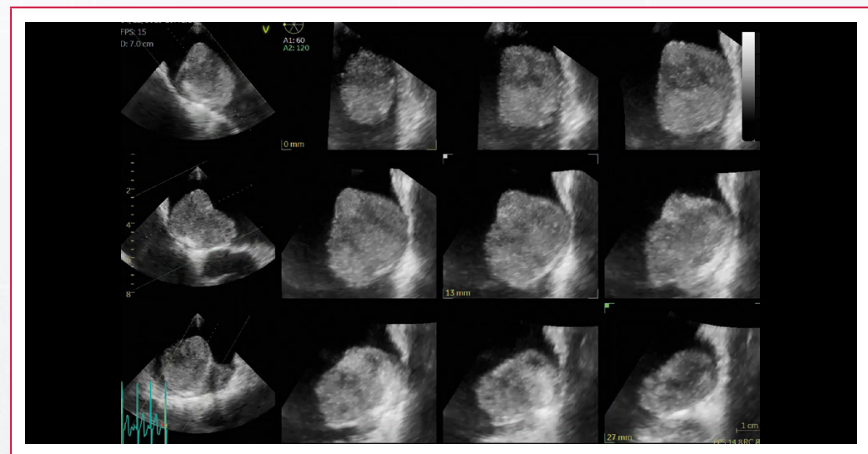

Vídeo 5. ETE. Volumen completo tridimensional (modalidad multicorte). Múltiples cortes tomográficos a través de la masa, en sentido lateral-medial y posterior-anterior. Nótese la heterogeneidad de la masa, según el corte que se visualice

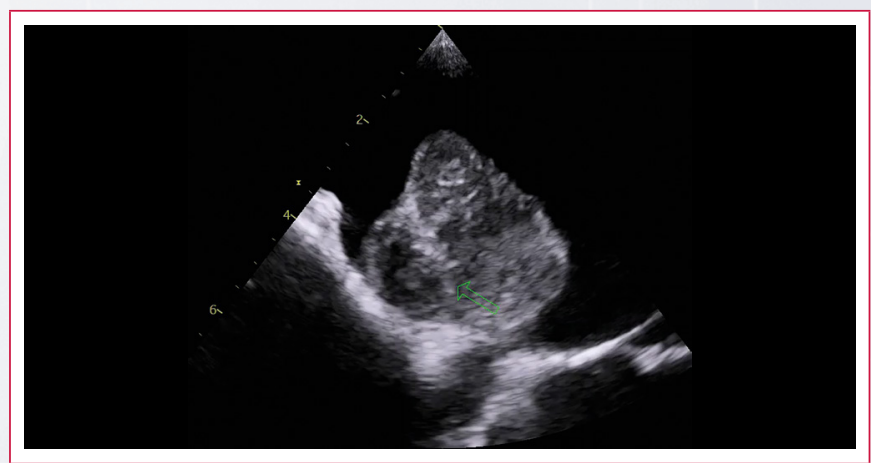

Vídeo 6. ETE bidimensional, Doppler color. Se observa la masa en aurícula izquierda, y el Doppler color (con límite de Nyquist de $16 \mathrm{~cm} / \mathrm{s}$ y optimización de ganancia) permite observar con claridad la presencia de flujo sanguíneo al interior de la masa (flecha verde) 


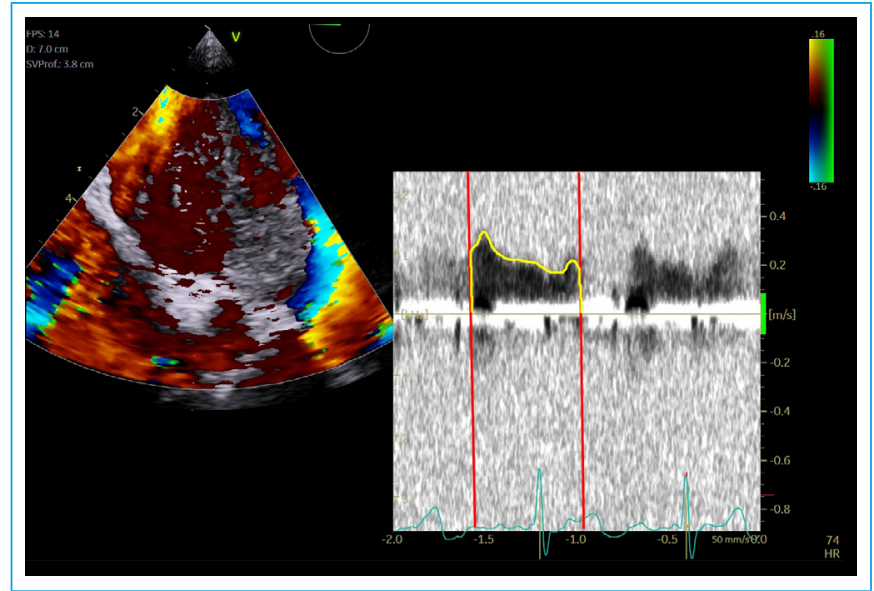

Figura 2 Doppler pulsado espectral. La muestra está colocada en el sitio donde se detectó el flujo sanguíneo en el interior de la masa; obsérvese la morfología típicamente coronaria del espectro (así como su predominancia diastólica), lo que confirma su origen

\section{Discusión}

El mixoma es el tumor cardíaco primario más común ${ }^{(1)}$. La mayoría de los casos son esporádicos y sus manifestaciones clínicas consisten en una tríada de síntomas constitucionales, embolización y obstrucción intracardíaca. Se localiza principalmente en la aurícula izquierda (75\%), en la aurícula derecha (23\%) y raramente en los ventrículos ${ }^{(2)}$. El diagnóstico usualmente no es difícil y, en general, se realiza a partir de ecocardiografía transtorácica/transesofágica. Sin embargo, y precisamente por esta posibilidad para hacer el diagnóstico con relativa facilidad, usualmente se dejan de lado algunas herramientas de la ecocardiografía que podrían complementar y/o afianzar el diagnóstico, lo que lleva en ocasiones a solicitar otras modalidades de imagen, que no en todos los casos son necesarias.

Se ha documentado que tanto la ecocardiografía transesofágica, como la ecocardiografía con contraste pueden confirmar la neovascularización de un mixoma $a^{(3,4)}$, lo que evidentemente confirma su naturaleza tumoral. De ahí la importancia de poder implementar sistemáticamente la búsqueda de flujo sanguíneo en el interior de cualquier masa intracardíaca con los parámetros del equipo adaptados para tal fin.

Por otra parte, la ecocardiografía tridimensional es otra de las herramientas que se deben utilizar en el abordaje diagnóstico de las masas cardíacas. Su capacidad de realizar reconstrucciones multiplanares permite una ubicación espacial exacta de la masa y, en el caso específico del mixoma, permite localizar con precisión el sitio de donde se origina el tumor ${ }^{(5,6)}$, hallazgo que no es tan factible con otras técnicas de imagen. Entre otras ventajas, esto permite un mejor abordaje y planeamiento quirúrgico.

\section{Conclusión}

La ecocardiografía, con sus diversas modalidades y técnicas, permite hacer diagnósticos más precisos y exactos de las masas cardíacas, y en particular del mixoma, si se aplican de forma sistemática.

\section{Ideas para recordar}

- La ecocardiografía tridimensional no es sólo una bonita imagen; incrementa la exactitud diagnóstica y permite un mejor planeamiento quirúrgico en el contexto de masas cardíacas.

- La visualización del flujo sanguíneo en el interior de una masa permite hacer el diagnóstico diferencial entre tumor y trombo; por tanto, se debe buscar intencionalmente este hallazgo con la ecocardiografía.

- La herramienta multicorte de la ecocardiografía tridimensional proporciona una detallada "disección anatómica" de las masas cardíacas (como el mixoma), permitiendo cierto grado de análisis tisular, lo que posibilita una mejor caracterización y acercamiento diagnóstico.

\section{Bibliografía}

1. Amano J, Kono T, Wada Y, et al. Cardiac myxoma: Its origin and tumor characteristics. Ann Thorac Cardiovasc Surg 2003; 9: 215-221.

2. Pepi M, Evangelista A, Nihoyannopoulos P, et al.; on behalf of the European Association of Echocardiography. Recommendations for echocardiography use in the diagnosis and management of cardiac sources of embolism. Eur J Echocardiogr 2010; 11 (6): 461-476.

3. Handke M, Goepfrich M, Keller H. Strongly neovascularized left atrial myxoma. Eur J Echocardiogr 2008; 9: 99-100.

4. Abdelmoneim SS, Beinier M, Dhoble A, et al. Assessment of the vascularity of a left atrial mass using myocardial perfusion contrast echocardiography. Echocardiography 2008; 25: 517-520.

5. Galzerano D, Pragliola C, Al Admawi M, et al. The role of 3D-echocardiographic imaging in the differential diagnosis of an atypical left atrial myxoma. Monaldi Archives for Chest Disease 2018; 88 (3).

6. Müller S, Feuchtner G, Bonatti J, et al. Value of transesophageal 3D echocardiography as an adjunct to conventional $2 \mathrm{D}$ imaging in preoperative evaluation of cardiac masses. Echocardiography 2008; 25: 624-631. 